\title{
Effect of Vancomycin Administration Method on Achieving and Maintaining Its Serum Concentrations In Intensive Care Patients
}

\author{
Vera Sokolova*, Olegs Sabelnikovs** \\ *University of Latvia, Riga, Latvia \\ **Department of Anaesthesia and Intensive Care, Riga Stradins University, Latvia
}

\begin{abstract}
Summary
Introduction. Vancomycin may be administered by bolus injections $(\mathrm{BI})$ and continuous infusion $(\mathrm{Cl})$. Data on $\mathrm{BI}$ and $\mathrm{Cl}$ clinical effectiveness and correlation with mortality, survival and renal injury is variable.
\end{abstract}

Aim of the study. The aim of the study was to compare the use of vancomycin continuous infusion and bolus administration in intensive care.

Materials and methods. Retrospective analysis of 46 ICU patients ( 23 patients received vancomycin $\mathrm{Cl}$ and $23-\mathrm{BI}$ ) was undertaken to compare efficacy and effect on mortality and morbidity of these two administration methods.

Results. The study revealed association of $\mathrm{Cl}$ administration with more effective $(15-25 \mathrm{mg} / \mathrm{L})$ serum vancomycin trough concentration maintenance $(31.2 \%$ vs $25.6 \%$ of treatment time, $\mathrm{p}=0.011)$ as well as with reduced incidence of subtherapeutic vancomycin levels $(34.3 \%$ vs $37.2 \%$ of treatment time, $p=0.015)$. We also observed better survival times using $\mathrm{Cl}$ mode.

Conclusions. $\mathrm{Cl}$ appears to be beneficial in terms of better maintenance of target VSTC and better patient survival. However, its use compared to bolus administration has no statistically significant impact on overall mortality and incidence of nephrotoxicity.

Key words: Vancomycin, continuous infusion vs intermittent, subtherapeutic concentrations, mortality, intensive care.

\section{INTRODUCTION}

Vancomycin is a glycopeptide antibiotic used for treatment of gram-positive (particularly MRSA) infections. It's characteristic features are narrow therapeutic index and time-dependent rather than concentration-dependent bactericidal effect. (2, 15, 16) Therefore maintaining certain serum trough concentrations is more important for clinical efficacy than reaching certain peak concentrations. (16) In order to improve clinical efficacy and reduce incidence of microorganism resistance in case of severe S.aureus infections maintenance of serum trough concentrations of $15-20 \mathrm{mg} / \mathrm{L}$ is recommended. $(15,16)$ Data suggesting a direct causal relationship between toxicity and specific vancomycin serum concentrations is limited; its evaluation is complicated by the presence of confounding nephrotoxic agents, variable definitions of toxicity, and inability to examine the time sequence of events surrounding changes in renal function secondary to vancomycin exposure. $(15,16)$

At P. Stradins Clinical University Hospital general Intensive Care Unit both vancomycin administration by bolus injections (using conventional doses of $1-2$ $\mathrm{g}$ once or twice daily, depending on vancomycin serum concentrations) and, recently, continuous infusion (over 24 hours using the adapted protocol of Sheffield Teaching Hospitals NHS Trust, United Kingdom, available for public review (7)) is used. The latter involves adjusted weight-specific loading dose administration immediately followed by continuous infusion, which rate is determined considering patient's renal function (urine output, creatinine clearance, serum urea and creatinine, use of renal replacement therapy). Subsequent CI rate adjustments are made daily according to the protocol depending on vancomycin serum trough concentrations (determined every morning and aiming $15-25 \mathrm{mg} / \mathrm{L}$ ).

\section{AIM OF THE STUDY}

The aim of the study was to compare the use of vancomycin continuous infusion and bolus administration in intensive care.

\section{MATERIALS AND METHODS}

Following approval by the University of Latvia Experimental and Clinical Medicine Institute Ethics Committee, a retrospective study of patients receiving vancomycin for treatment of gram-positive infections was carried out to compare BI and CI administration methods. The study involved case note analysis of randomly selected patients who received vancomycin on our ICU between January and April 2013.

Clinical and demographic data on 46 patients $(23$ received vancomycin by CI, 23 - by BI) were gathered, including demographics (age, sex), diagnosis, surgeries performed, ICU length of stay, length of artificial ventilation, as well as length of vancomycin therapy, 24 hour doses used, frequency of administration (for $\mathrm{BI}$ ) and administration rate (for CI), dynamics of daily determined vancomycin serum trough concentrations (VSTC), renal function dynamics (urine output, creatinine clearance, serum urea and creatinine), presence of renal replacement therapy, and clinical outcome (transfer to general ward or death). 
CI and BI administration methods were compared in terms of the following:

1) speed of achievement of therapeutically effective VSTC (time in days since commencement of vancomycin therapy till trough concentration of $>15 \mathrm{mg} / \mathrm{L}$ achieved for the first time);

2) effectiveness for maintenance of target VSTC: absolute (amount of therapy days when VSTC was within the target range) and relative time (percentage of days when VSTC was within the target range out of the full length of therapy [in days]);

3) frequency of subtherapeutic and supratherapeutic VSTC episodes for CI and BI;

4) Differences in patient overall ICU mortality, survival time and development of nephrotoxicity.

Data were analyzed and statistically processed using SPSS Statistics 20 software.

\section{RESULTS}

Age structure of the patients in CI and BI groups (Figure 1 ) is comparable within the $41-80$ y.o. range; however, BI group had more patients $18-40$ y.o. and no patients $>$ 80 y.o.

Length of vancomycin therapy varied between 3 and 21 days: mean $9.74 \pm 4.67$ days in CI group and $6.17 \pm 3.84$ days in BI group. Mean ICU stay was 27.65 days in CI group and 19.91 days in BI group.

Overall ICU mortality was $30.43 \%$ (14 patients): 9 patients $(39.13 \%)$ in CI group and 5 patients $(21.74 \%)$ in BI group. Analysis of patient mortality with Fisher test showed no statistically significant association with the use of CI (OR 1.8; $p=0.337)$. A possible cause of 1.8 fold higher mortality in CI group could be heterogeneity of the study population in terms of diagnosis, age, co-morbidities and baseline physiological state; it is also possible that clinicians were choosing CI for more severely ill patients with initially known worse prognosis.

Analysis of CI and BI groups using Kaplan-Meier survival curves showed statistically significant better survival in CI group (Figure 2), e.g. 5-day survival rate was $50 \%$ in CI group vs $15 \%$ in BI group $(p=0.005$ with Mantel-Cox test).

Speed of achievement of therapeutically effective VSTC was faster in CI group, however analysis of the data showed that these results are not statistically significant: CI group $3^{\mathrm{d}}[2-4]$ vs BI group $4^{\text {th }}[2-4]$ treatment days ( $p=0.391$ with Mann-Whitney U test). Maintenance of target VSTC $(15-25 \mathrm{mg} / \mathrm{L})$ was better using CI (3.7 \pm 3.4 days or $47.6 \pm 31.2 \%$ of treatment time) than BI $(1.4 \pm 1.6$ days or $25.6 \pm 26.8 \%$ of treatment time). Analysis with Mann-Whitney U test proved statistical significance of these results $(p=0.011)$. Frequency of subtherapeutic VSTC episodes was higher in BI group - mean $3.1 \pm 1.95$ days $(66.4 \pm 34.3 \%$ of treatment time) versus $2.1 \pm 2.21$ days $(38.7 \pm 37.2 \%$ of treatment time) in CI group. Data analysis with Mann-Whitney U test showed statistically significant lower incidence of subtherapeutic VSTC in CI group $(\mathrm{p}=$ $0.015 ; 25[0-71]$ vs 75 [38-100]).

Frequency of supratherapeutic VSTC episodes was almost 2 times higher in CI group - mean $1.3 \pm 1.68$ days $(17.3 \pm 24.89 \%$ of treatment time) versus $0.4 \pm$ 7.22 days $(8.1 \pm 17.38 \%$ of treatment time) in BI group. However, analysis with Mann-Whitney U test showed absence of statistical significance of these data $(\mathrm{p}=$ 0.092; $(10$ [0 - 29] vs $0[0-15])$, which means that it cannot be extrapolated to a larger population.

Renal function dynamics were assessed using daily serum creatinine values and the following definition of vancomycin induced nephrotoxicity: serum creatinine increase by $\geq 44,2 \mathrm{mcmol} / \mathrm{L}$ (or $\geq 50 \%$ over baseline) in consecutively obtained daily serum creatinine values or a drop in calculated CLcr of $50 \%$ from baseline on two consecutive days in the absence of an alternative explanation. $(4,6,8,9,10,17)$ Patients with initially high baseline serum creatinine levels (>113 mcmol/L) were excluded from further analysis. Due to retrospective nature of our study only serum creatinine levels and not creatinine clearance criterion was used. Figure 3 reflects the incidence of serum creatinine level dynamics corresponding to the above definition of nephrotoxicity in both groups.

Incidence of nephrotoxicity in CI group was 7.1\% (1 of 14 patients), but in BI group - 25\% (4 of 16 patients). However, analysis with Fisher test shows absence of statistical significance of these data $(p=0.336)$.

\section{DISCUSSION}

Our study results show absence of statistically significant difference in patient mortality between vancomycin CI and BI groups. This is similar to the data from the majority of other studies investigating this correlation. (1, 3, 15, 16, 18, 19) However, one matched cohort study reported lower mortality rates for critically ill patients with ventilator-associated pneumonia receiving vancomycin in CI $(25 \%$ vs $55 \%)$. Multiple regression analysis of the data confirmed that CI was associated with improved survival. (12) The latter corresponds to our results which also show improved survival in CI group.

Faster achievement of effective VSTC which was seen in our study is similar to the data from other scientists $(5,11,13,14,18)$, however our results on this do not appear to be statistically significant.

As regards CI superiority in terms of lower VSTC variability and its better maintenance within the target range our results match the data of other published studies. (11, 13, 14, 18, 19)

Our study didn't reveal correlation between CI use and lower incidence of nephrotoxicity. We find this difficult to investigate due to wide ICU patient heterogeneity and specific features, such as variable co-morbidities, hemodynamic instability, 24-hour urine output changes, body fluid compartment volume shifts, and therapeutic use of other potentially nephrotoxic agents, which makes it impossible to attribute serum creatinine changes solely to the effect of vancomycin. 


\section{CONCLUSIONS}

We found the use of vancomycin CI protocol associated with more straightforward and uniform action plan for clinicians, as it provides a simple tool for dose/rate adjustment judging by the daily VSTC measurements and other parameters.

Continuous infusion appears to be beneficial in terms of better maintenance of target VSTC and better patient survival. However, its use compared to bolus administration has no statistically significant impact on overall mortality and incidence of nephrotoxicity.

\section{Conflict of interest: None}

\section{REFERENCES}

1. Cataldo MA et al. Continuous versus intermittent infusion of vancomycin for the treatment of Gram-positive infections, systematic review and meta-analysis. // J Antimicrob Chemother, 2012; 67(1):17-24.

2. Devabhakthuni S. Antibiotic Pharmacokinetic Monitoring. // Published 2011. Retrieved on May 22, 2013 from www.ashp.org/DocLibrary/ MemberCenter/NPF/2011Pearls/ AntibioticPharmacokinetic-Monitoring.aspx

3. Di Filippo A et al. Continuous infusion of vancomycin in methicillin-resistant staphylococcus infection. // Chemotherapy, 1998; 44(1):63-68.

4. Hidayat LK et al. High-dose vancomycin therapy for methicillin-resistant Staphylococcus aureus infections, efficacy and toxicity. // Arch Intern Med, 2006; 166:2138-2144.

5. Ibrahim NS et al. Time to Achieving Target Concentration of Vancomycin, Intermittent Infusion versus Continuous Infusion of Vancomycin in Intensive Care Unit. // Published 2011 . Retrieved on March 22, 2013 from http, //www.kck.usm.my/ husm/pharmacy/images/ penerbitan

6. Jeffres MN et al. A retrospective analysis of possible renal toxicity associated with vancomycin in patients with healthcare-associated methicillinresistant Staphylococcus aureus pneumonia. // Clin Ther, 2007; 29:1107-1115.

7. Law Min C, Bourne R. Continuous Vancomycin Infusion Guidelines in Critical Care. // Sheffield Teaching Hospitals NHS Foundation Trust Guidelines, 2012.

8. Lodise TP et al. Larger vancomycin doses $(\geq 4$ grams/day) are associated with an increased incidence of nephrotoxicity. // Antimicrob Agents Chemotherapy, 2008; 52:1330-1336.

9. Nguyen $M$ et al. Nephrotoxicity associated with high dose vs standard dose vancomycin therapy. // In: 47th Interscience Conference on Antimicrob Agents Chemotherapy. 2007; Chicago, IL, September 2007.
10. Pauly DJ et al. Risk of nephrotoxicity with combination vancomycin-aminoglycoside antibiotic therapy. // Pharmacotherapy, 1990; 10:378-382.

11. Pea $F$ et al. Prospectively validated dosing nomograms for maximizing pharmacodynamics of vancomycin administered by continuous infusion in critically ill patients. // Antimicrob Agents Chemotherapy, 2009; 53(5):1863-1867.

12. Rello J et al. Pneumonia caused by oxacillinresistant Staphylococcus aureus treated with glycopeptides. // Crit Care Med, 2005; 33(9):19831987.

13. Roberts JA et al. Better outcomes through continuous infusion of time dependent antibiotics to critically ill patients? // Curr Opin Crit Care, 2008; 14:390-396.

14. Roberts JA, Lipman J. Antibacterial dosing in intensive care, pharmacokinetics, degree of disease and pharmacodynamics of sepsis. // Clin Pharmacokinet, 2006; 45:755-773.

15. Rybak MJ et al. Vancomycin Therapeutic Guidelines, A Summary of Consensus Recommendations from the Infectious Diseases Society of America, the American Society of Health-System Pharmacists, and the Society of Infectious Diseases Pharmacists. // Clin Infect Dis, 2009; 49:325-327.

16. Rybak MJ, Lomaestro B. Therapeutic monitoring of vancomycin in adult patients, A consensus review of the American Society of Health-System Pharmacists, the Infectious Diseases Society of America, and the Society of Infectious Diseases Pharmacists. // AJHP, 2009; 66:82 - 98.

17. Rybak MJ et al. Nephrotoxicity of vancomycin alone and with an aminoglycoside. // J Antimicrob Chemother, 1990; 25:679-687.

18. Vuagnat A et al. High dose vancomycin for osteomyelitis, continuous vs intermittent infusion. // J Clin Pharm Ther, 2004; 29(4):351-357.

19. Wysocki $\mathrm{M}$ et al. Continuous versus intermittent infusion of vancomycin in severe Staphylococcal infections, prospective multicenter randomized study. // Antimicrob Agents Chemotherapy, 2001; 45(9):2460-2467.

\author{
Address: \\ Vera Sokolova \\ 16 Leven drive, Bearsden \\ Glasgow, \\ United Kingdom \\ G61 2EE \\ E-mail: reflecting_soul@inbox.lv
}




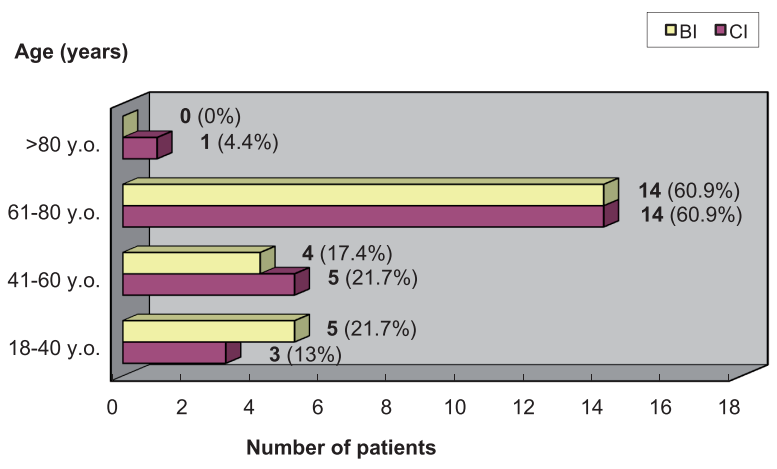

Fig. 1. Patient's age structure

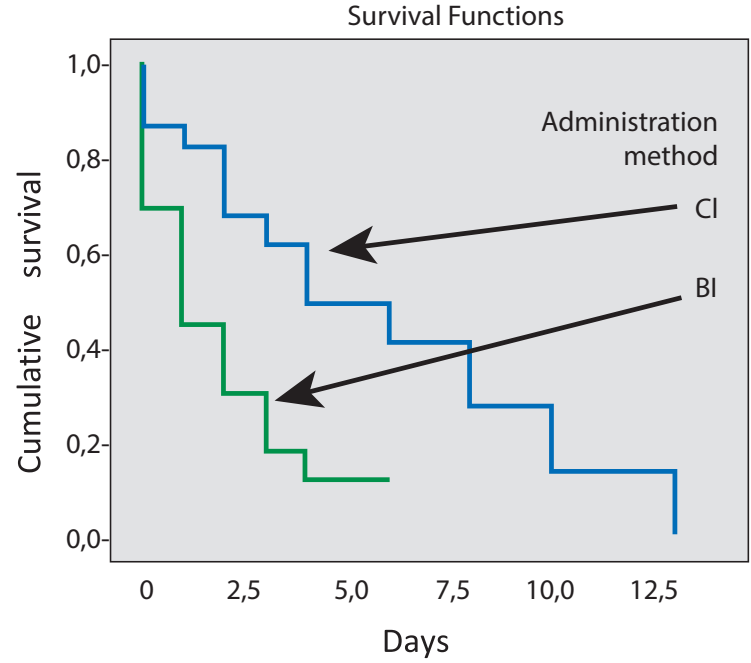

Fig. 2. Patient's survival in CI and BI groups

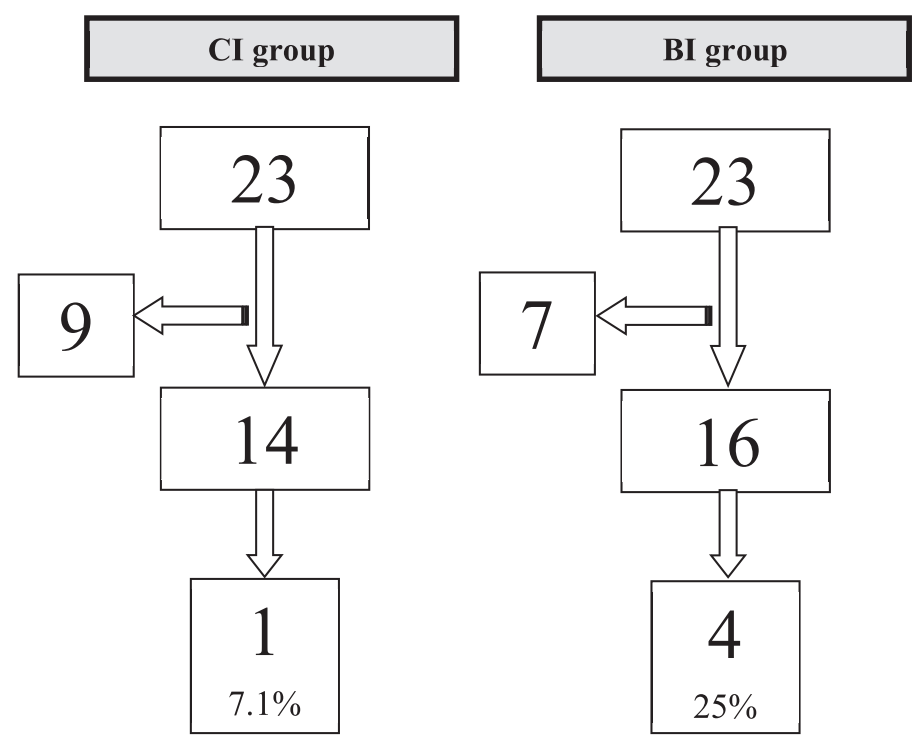

Total amount of patients in the group

Exclusion of patients with initially high baseline serum creatinine $(>113 \mathrm{mcmol} / \mathrm{L})$

Number of further analysed patients

Number of patients in each group in whom serum creatinine rise corresponding to the used nephrotoxicity criterion was observed

Fig. 3. Evaluation of serum creatinine level dynamics 